\title{
Marek Nasieniewski
}

\section{A RELATIONAL SYLLOGISTIC}

\section{Introduction}

In [1] J. Perzanowski formulated, among others, an ontology expressed in the relational language. He presented some interesting connections which hold between these relations. In the present paper we focus on further analysis of these relations.

Let us consider a binary relation $E$. We recall the following Perzanowski's definitions:

$$
\begin{aligned}
& \text { " } x \text { is a part of } y \text { ": } \\
& x P y \leftrightarrow \bigwedge_{z}(z E x \rightarrow z E y) \\
& \text { (C) “ } x \text { is covered by } y " \text { : } \\
& x C y \leftrightarrow \bigwedge_{z}(y E z \rightarrow x E z) \\
& x H y \leftrightarrow \bigwedge(z E x \rightarrow y E z) \\
& \text { (D) " } x \text { dominates } y " \text { ": } \\
& x D y \leftrightarrow \bigwedge_{z}(y E z \rightarrow z E x) \\
& x L y \leftrightarrow \bigwedge_{z}(z P x \rightarrow z E y) \\
& x A y \leftrightarrow \bigwedge_{z}(y C z \rightarrow x E z)
\end{aligned}
$$$$
\text { (H) " } x \text { houses } y \text { ": }
$$$$
\text { (L) " } x \text { is located in } y " \text { " }
$$

(A) " $x$ is allocated in $y "$ :

To any formula of the following form:

$$
\bigwedge_{x} \bigwedge_{y} \bigwedge_{z}(x X y \wedge y Y z \rightarrow x Z z)
$$


where $X, Y, Z \in\{E, P, C, H, D, L, A\}^{1}$, there corresponds a syllogism of the first figure $^{2}$ :

$$
\begin{gathered}
y Y z \\
x X y \\
\hline x Z z
\end{gathered}
$$

and there is ono-to-one correspondence between tautologies of this form and valid syllogisms of the first figure, where validity is understood as explained below.

Definition 1. A syllogism

$$
\begin{aligned}
& y Y z \\
& x X y \\
& \hline x Z z
\end{aligned}
$$

where $X, Y, Z \in\{E, P, C, H, D, L, A\}$, is valid iff for any set $U$, any 2-ary relation $E$ on $U$ and any $x, y, z \in U$ the following condition holds: if $\langle x, y\rangle \in X,\langle y, z\rangle \in Y$, then $\langle x, z\rangle \in Z$, where $X, Y$ and $Z$ are equal to the relation $E$ or are respective relations defined by the conditions $(\mathbf{P}),(\mathbf{C}),(\mathbf{H}),(\mathbf{D}),(\mathbf{L}),(\mathbf{A})$.

Equivalently, the above defined syllogisms can be treated as inclusions of the form:

$$
X \circ Y \subseteq Z
$$

holding for respective relations.

One can easily see that there is $7^{3}=343$ syllogisms of the first figure. We will indicate all valid syllogisms.

\section{Valid syllogisms}

By first-order logic we see that for any initial relation $E$, the relations $P$ and $C$ are preorders i.e. are reflexive and transitive ${ }^{3}$ :

$$
\begin{gathered}
x P x \\
x P y \wedge y P z \rightarrow x P z \\
x C x \\
x C y \wedge y C z \rightarrow x C z
\end{gathered}
$$

${ }^{1}$ Formally speaking, we should differentiate between relations, corresponding constants of the first order language and logical constants of the language of syllogistic. The context will disambiguate the meaning of a given symbol.

${ }^{2}$ Similarly, by considering converses of these relations we can obtain syllogisms of other figures.

${ }^{3} \mathrm{We}$ will omit general quantifiers. 
We omit proofs of some other theorems which have been also proved in [1]:

$$
\begin{aligned}
& x A y \wedge y C z \rightarrow x A z \\
& x C y \wedge y A z \rightarrow x A z \\
& x A y \wedge y C z \rightarrow x E z \\
& x C y \wedge y A z \rightarrow x E z \\
& x P y \wedge y L z \rightarrow x L z \\
& x P y \wedge y L z \rightarrow x E z \\
& x L y \wedge y P z \rightarrow x L z \\
& x L y \wedge y P z \rightarrow x E z \\
& x E y \wedge y P z \rightarrow x E z \\
& x C y \wedge y E z \rightarrow x E z
\end{aligned}
$$

We let ourselves include proofs of theorems which were not presented in [1]:

$$
x A y \wedge y P z \rightarrow x E z
$$

Proof. Let's fix some set $U^{4}$. Assume that for $x, y, z \in U$, it holds that $x A y \wedge y P z$. We show that $x E z$. By (\$) we have that $y C y$, by the definition (A) we obtain that $\bigwedge_{w}(y C w \rightarrow x E w)$, thus in particular we have $y C y \rightarrow x E y$, i.e. $x E y$. By the definition of the relation $P$ we have $\bigwedge_{w}(w E y \rightarrow w E z)$ and once again if $w$ equals $x$ we receive $x E y \rightarrow x E z$. Thus, since $x E y$, we have also $x E z$.

$$
x C y \wedge y L z \rightarrow x E z
$$

Proof. Let's assume that for $x, y, z \in U$, it holds that $x C y \wedge y L z$. We prove that $x E z$. By the definition $(\mathbf{L})$, we receive that $\bigwedge_{w}(w P y \rightarrow w E z)$, in particular we have $y P y \rightarrow y E z$, thus, by $(\dagger)$ we obtain $y E z$. By the definition (C) via the assumption $x C y$ we have that $\bigwedge_{w}(y E w \rightarrow x E w)$ and thus we receive $y E z \rightarrow x E z$, and since $y E z$, so also $x E z$.

$$
x P y \wedge y H z \rightarrow x H z
$$

Proof. Let's assume that for $x, y, z \in U$, it holds that $x P y \wedge y H z$. We prove that $x H z$. To show this let's take any $u \in U$, and assume that $u E x$. We prove that $z E u$. By the definition (P) we have $\bigwedge_{w}(w E x \rightarrow w E y)$ and in particular we conclude that $u E x \rightarrow u E y$, i.e. $u E y$. By the definition $(\mathbf{H})$ we receive $\bigwedge_{w}(w E y \rightarrow z E w)$ and we have $u E y \rightarrow z E u$, but since $u E y$, we also have $z E u$.

\footnotetext{
${ }^{4}$ In the remaining proofs such assumption will be taken for granted.
} 


$$
x D y \wedge y C z \rightarrow x D z
$$

Proof. Let's assume that for some $x, y, z \in U$, it holds that $x D y \wedge y C z$. We prove that $x D z$. Let's take any $u \in U$ and assume that $z E u$. We prove that $u E x$. By the definition (C) we have $\bigwedge_{w}(z E w \rightarrow y E w)$ in particular we have $z E u \rightarrow y E u$, thus $y E u$. By the definition (D) we obtain $\bigwedge_{w}(y E w \rightarrow w E x)$, and therefore we have $y E u \rightarrow u E x$, however, since $y E u$, thus also $u E x$.

\section{Refuted syllogisms}

From the list of all possible syllogisms of the first figure one have to reject quite a lot of them. We will indicate $E$ being a counterexample for a possible validity of a given syllogism.

Remark 1. For the relation $E \subseteq U^{2}$, where $U=\{a\}$ and $E=\varnothing$ it holds that: $X \circ Y \nsubseteq Z$, where $X, Y \in\{P, C, D, H\}$, while $Z \in\{E, L, A\}$.

Remark 2. For the relation $E \subseteq U^{2}$, where $U=\{a, b\}$ and $E=\{\langle a, a\rangle\}$ it holds that: $E \circ Y \nsubseteq Z$, where $Y \in\{P, C, H, D\}$, while $Z \in\{A, L\}$,

$X \circ E \nsubseteq Z$, where $X \in\{P, C, H, D\}$, while $Z \in\{A, L\}$,

$C \circ P \nsubseteq Z$, where $Z \in\{P, C, H, D\}$, $P \circ C \nsubseteq Z$, where $Z \in\{P, C, H, D\}$, $P \circ D \nsubseteq Z$, where $Z \in\{P, C, H, D\}$, $D \circ P \nsubseteq Z$, where $Z \in\{P, C, H, D\}$, $C \circ H \nsubseteq Z$, where $Z \in\{P, C, H, D\}$, $H \circ C \nsubseteq Z$, where $Z \in\{P, C, H, D\}$, $C \circ C \nsubseteq Z$, where $Z \in\{P, H\}$, $C \circ D \nsubseteq Z$, where $Z \in\{P, H\}$, $D \circ C \nsubseteq Z$, where $Z \in\{P, H\}$, $P \circ P \nsubseteq Z$, where $Z \in\{C, D\}$, $P \circ E \nsubseteq E$, $E \circ C \nsubseteq E$, $H \circ E \nsubseteq E$.

Remark 3. For the relation $E \subseteq U^{2}$, where $U=\{a, b\}$ and $E=\{\langle a, b\rangle,\langle b, a\rangle\}$ it holds that:

$E \circ Y \nsubseteq Z$, where $Y, Z \in\{P, C, H, D\}$,

$X \circ E \nsubseteq Z$, where $X, Z \in\{P, C, H, D\}$,

$E \circ A \nsubseteq Z$, where $Z \in\{A, L, E\}$,

$P \circ A \nsubseteq Z$, where $Z \in\{P, H\}$,

$A \circ Y \nsubseteq Z$, where $Y \in\{P, C, H\}$, while $Z \in\{P, C, H\}$, 
$X \circ A \nsubseteq Z$, where $X \in\{C, H\}$, while $Z \in\{P, H\}$,

$C \circ A \nsubseteq C$,

$A \circ E \nsubseteq Z$, where $Z \in\{A, L, E\}$,

$L \circ H \nsubseteq H$.

Remark 4. For the relation $E \subseteq U^{2}$, where $U=\{a, b\}$ and $E=\{\langle a, a\rangle,\langle a, b\rangle\}$ it holds that:

$P \circ A \nsubseteq Z$, where $Z \in\{C, D, A, L, E\}$,

$H \circ A \nsubseteq Z$, where $Z \in\{C, D, A, L, E\}$,

$A \circ P \nsubseteq Z$, where $Z \in\{D, L\}$,

$C \circ A \nsubseteq Z$, where $Z \in\{D, L\}$,

$A \circ C \nsubseteq Z$, where $Z \in\{D, L\}$,

$A \circ H \nsubseteq Z$, where $Z \in\{D, L\}$,

$C \circ C \nsubseteq D$,

$P \circ P \nsubseteq H$.

Remark 5. For the relation $E \subseteq U^{2}$, where $U=\{a, b\}$ and $E=\{\langle a, a\rangle,\langle a, b\rangle,\langle b, b\rangle\}$ it holds that:

$H \circ L \nsubseteq Z$, where $Z \in\{P, C, D, H, E, L, A\}$,

$L \circ H \nsubseteq Z$, where $Z \in\{P, C, D, E, L, A\}$,

$H \circ P \nsubseteq Z$, where $Z \in\{P, C, H, D\}$,

$P \circ H \nsubseteq Z$, where $Z \in\{P, C, D\}$,

$C \circ D \nsubseteq Z$, where $Z \in\{C, D\}$,

$D \circ C \nsubseteq C$,

$A \circ H \nsubseteq Z$, where $Z \in\{A, E\}$.

Remark 6. For the relation $E \subseteq U^{2}$, where $U=\{a, b\}$ and $E=\{\langle a, a\rangle,\langle b, a\rangle,\langle b, b\rangle\}$ it holds that:

$L \circ D \nsubseteq Z$, where $Z \in\{P, C, H, D, L, A, E\}$,

$E \circ H \nsubseteq E$,

$D \circ E \nsubseteq E$,

$E \circ D \nsubseteq E$.

Remark 7. For the relation $E \subseteq U^{2}$, where $U=\{a, b, c\}$ and $E=\{\langle a, b\rangle,\langle b, c\rangle\langle c, a\rangle\}$ it holds that:

$X \circ Y \nsubseteq Z$, where $X, Y \in\{H, D\}$, while $Z \in\{P, C, H, D\}$,

$X \circ A \nsubseteq Z$, where $X \in\{D, L, A\}$, while $Z \in\{E, P, C, H, D, L, A\}$,

$A \circ Y \nsubseteq Z$, where $Y \in\{D, L\}$, while $Z \in\{E, P, C, H, D, L, A\}$,

$E \circ E \nsubseteq Z$, where $Z \in\{L, A\}$,

$E \circ L \nsubseteq Z$, where $Z \in\{E, P, C, H, D, L, A\}$,

$L \circ E \nsubseteq Z$, where $Z \in\{E, P, C, H, D, L, A\}$,

$L \circ L \nsubseteq Z$, where $Z \in\{E, P, C, H, D, L, A\}$. 
Remark 8. For the relation $E \subseteq U^{2}$, where $U=\{a, b, c\}$ and $E=\{\langle a, a\rangle,\langle b, a\rangle$, $\langle b, b\rangle,\langle c, b\rangle,\langle c, c\rangle\}$ it holds that:

$P \circ L \nsubseteq A$,

$X \circ P \nsubseteq A$, where $X \in\{L, A\}$.

$E \circ Y \nsubseteq Z$, where $Y \in\{E, A\}$ while $Z \in\{P, C, H, D\}$.

$A \circ E \nsubseteq Z$, where $Z \in\{P, C, H, D\}$.

$C \circ L \nsubseteq Z$, where $Z \in\{P, C, H, D, L, A\}$.

$D \circ L \nsubseteq Z$, where $Z \in\{P, C, H, D, L, A, E\}$.

$E \circ E \nsubseteq E$,

$L \circ C \nsubseteq Z$, where $Z \in\{E, P, C, H, D, L, A\}$.

Remark 9. For the relation $E \subseteq U^{2}$, where $U=\{a, b, c, d\}$ and $E=\{\langle a, a\rangle,\langle a, b\rangle$, $\langle b, b\rangle,\langle b, c\rangle,\langle c, c\rangle,\langle c, d\rangle,\langle d, d\rangle\}$ it holds that:

$P \circ L \nsubseteq Z$, where $Z \in\{P, C, H, D\}$,

$L \circ P \nsubseteq Z$, where $Z \in\{P, C, H, D\}$.

\section{Generalized syllogisms}

Definition 2. By a generalized syllogism we mean a pair $\left\langle\left\langle X_{1}, \ldots, X_{n}\right\rangle, X\right\rangle$, where $n \geq 2, X_{1}, \ldots, X_{n}, X \in\{E, P, C, H, D, L, A\}$.

The syllogisms falling under the above definition can be treated as sequents. Farther in the paper we will use a notion of a proof for the sequent calculus.

We consider the following version of a cut rule:

(Cut)

$$
\frac{\left\langle\left\langle X_{1}, \ldots, X_{i}, \ldots, X_{n}\right\rangle, X\right\rangle,\left\langle\left\langle Y_{1}, \ldots, Y_{m}\right\rangle, X_{i}\right\rangle}{\left\langle\left\langle X_{1}, \ldots, X_{i-1}, Y_{1}, \ldots, Y_{m}, X_{i+1}, \ldots, X_{n}\right\rangle, X\right\rangle}
$$

Definition 3. We say that a generalized syllogism $\left\langle\left\langle X_{1}, \ldots, X_{n}\right\rangle, X_{n}\right\rangle$ is provable iff there is a proof of $\left\langle\left\langle X_{1}, \ldots, X_{n}\right\rangle, X_{n}\right\rangle$ in the sense of sequent calculus, where the axioms are

1. $\langle\langle P, P\rangle, P\rangle$

2. $\langle\langle C, C\rangle, C\rangle$

3. $\langle\langle A, P\rangle, E\rangle$

4. $\langle\langle C, L\rangle, E\rangle$

5. $\langle\langle P, H\rangle, H\rangle$

6. $\langle\langle D, C\rangle, D\rangle$

7. $\langle\langle A, C\rangle, A\rangle$

8. $\langle\langle C, A\rangle, A\rangle$ 
9. $\langle\langle A, C\rangle, E\rangle$

10. $\langle\langle C, A\rangle, E\rangle$

11. $\langle\langle P, L\rangle, L\rangle$

12. $\langle\langle P, L\rangle, E\rangle$

13. $\langle\langle L, P\rangle, L\rangle$

14. $\langle\langle L, P\rangle, E\rangle$

15. $\langle\langle E, P\rangle, E\rangle$

16. $\langle\langle C, E\rangle, E\rangle$

and (Cut) is the only rule of inference.

Definition 4. A generalized syllogism $\left\langle\left\langle X_{1}, \ldots, X_{n}\right\rangle, X\right\rangle$ is valid iff for any set $U$, any 2-ary relation $E$ on $U$ and any elements $x_{0}, x_{1}, \ldots, x_{n} \in U$ it holds that: if $\left\langle x_{0}, x_{1}\right\rangle \in X_{1},\left\langle x_{1}, x_{2}\right\rangle \in X_{2}, \ldots,\left\langle x_{n-1}, x_{n}\right\rangle \in X_{n}$, then $\left\langle x_{0}, x_{n}\right\rangle \in X$, where $X_{1}$, $X_{2}, \ldots, X_{n}$ are equal to the relation $E$ or are respective relations defined by the conditions $(\mathbf{P}),(\mathbf{C}),(\mathbf{H}),(\mathbf{D}),(\mathbf{L}),(\mathbf{A})$.

By previous observations we have

Lemma. Axioms 1-16 are valid.

Since the cut rule saves validity of syllogisms we have:

Theorem. For any generalized syllogism $\mathcal{S}$, if $\mathcal{S}$ is provable, then $\mathcal{S}$ is valid.

\section{References}

[1] Perzanowski, J., "Locative Ontology. Parts I-III", Logic and Logical Philosophy 1 (1993): 7-94.

Marek Nasieniewski

Nicolaus Copernicus University

Department of Logic and Semiotics

ul. Asnyka 2

PL 87-100 Toruń, Poland

mnasien@uni .torun.pl 